\title{
Thrombophilias: therapeutic employment of direct oral anticoagulants in venous hypercoagulable states
}

\author{
Federico Cacciapuoti \\ Department of Internal Medicine, Luigi Vanvitelli Campania University, Napoli, Italy
}

\begin{abstract}
Thrombophilia or hypercoagulable state is a predisposition to form clots. Thrombophilia can be inherited or acquired, and prevalently involves venous vessels. Inherited thrombophilia consists of congenital conditions, as methylenetetrahydrofolate reductase polymorphism, Factor V Leiden and prothrombin gene mutations, natural anticoagulant deficiencies, high level of factor VIII, or dysfibrinogenemia. These congenital disorders can be responsible for venous thromboembolism, particularly deep venous thrombosis, pulmonary embolism, and, less frequently, mesenteric veins thrombosis, kidneys' veins thrombosis or retinal vein occlusion. Acquired thrombophilia can be associated both with venous and arterial thrombosis and may be caused by antiphospholipid syndrome, aging, some malignancies, oral contraceptive use, heparin-induced thrombocytopenia, and human immunodeficiency virus. Antiplatelets' drugs are employed in arterial thrombosis, while, heparins/oral vitamin K antagonists are indicated for acute and long-term anticoagulation. However, new oral anticoagulants can be usefully used for venous thromboembolic events. Recent experiences demonstrated that their employment is useful in some thrombophilias only, whereas other investigations are requested to evaluate their use in all hypercoagulable disorders.
\end{abstract}

\section{Thrombophilia}

The prevalence of coagulant on anticoagulant factors causes an impaired hemostatic balance, named as thrombophilia. ${ }^{1-3}$ This can be a hereditary or acquired disorder, dependent on an abnormal increase to form a blood clot and higher than average risk of thrombosis. ${ }^{4}$ Acquired thrombophilia is due to the antiphos-

Correspondence: Federico Cacciapuoti, Department of Internal Medicine, Luigi Vanvitelli Campania University, piazza L. Miraglia 2, Napoli, Italy

Tel.: +39.081.566502.

E-mail: fulviocacciapuoti@gmail.com

Key words: Thrombophilia; venous thrombosis; arterial thrombosis; direct oral anticoagulants.

Conflict of interests: the author declares no potential conflict of interests.

Ethical approval: this article does not contain any studies with human participants or animals.

Received for publication: 16 April 2020.

Revision received: 5 June 2020

Accepted for publication: 15 June 2020.

This work is licensed under a Creative Commons Attribution NonCommercial 4.0 License (CC BY-NC 4.0).

${ }^{\circ}$ Copyright: the Author(s), 2020

Licensee PAGEPress, Italy

Italian Journal of Medicine 2020; 14:136-142

doi:10.4081/itjm.2020.1296 pholipid syndrome, advancing age, some malignancies, oral contraceptive drugs, heparin-induced thrombocytopenia, human immunodeficiency virus, polycythemia, and others. ${ }^{5,6}$ On the contrary, hereditary thrombophilia happens when some congenital abnormalities such as gene polymorphism of the enzyme methylene-tetrahydrofolate reductase (MTHFR), Factor $\mathrm{V}$ Leiden or prothrombin gene mutations, antithrombin III, protein $\mathrm{C}$, or protein $\mathrm{S}$ deficiencies, elevation of factor VIII, or dysfibrinogenemia, are present. $^{7}$

Apart from the acquired and congenital thrombophilias, we divide hypercoagulable states into venous and arterial thrombophilia, according to the tract of circulation involved. The former is defined as a predisposition to form clots, which can mainly involve venous vessels. ${ }^{8,9}$ Instead, arterial thrombophilia includes thrombosis in arterial vessels.

In this report, we defined the different forms of thrombophilia reported to the etiology (congenital or acquired) and location (arterial and vein) and their causes and proposed the employment of direct oral anticoagulants (DOACs) instead of vitamin $\mathrm{K}$ antagonists (VKAs) in some venous hypercoagulable states.

\section{Inherited thrombophilia}

This condition is defined as genetic predisposition to form thrombi prevalently in the venous vessels. The leading causes responsible for the inherited hypercoagulable state are reported in Table 1. 


\section{Homocysteine}

Homocysteine (Hcy) is a sulfhydryl-containing amino acid synthesized during the Methionine-metabolism. Two pathways control the serum level of Hcy: remethylation and trans-sulfuration, respectively involving the enzyme MTHFR and cystathionine- $\beta$-synthase. ${ }^{10,11}$ We only illustrate the impaired remethylation pathway, responsible for inherited mild hyperhomocysteinemia (HHcy), due to the gene mutation of the enzyme MTHFR. In homozygous trait, the MTHFR mutation reduces its activity by $50-60 \%$, while the enzymatic activity of heterozygous trait is more modestly reduced. ${ }^{12}$

Accumulating evidences link HHcy levels to thrombosis both in arterial and venous vessels. The role of Hcy in venous thromboembolism has been studied less extensively than its role in arterial diseases, and nowadays, it seems quite controversial. It is also possible that HHcy plays a role in the pathogenesis of venous thromboembolism only as an additional risk factor in the presence of other thrombophilic disorders. Among these, Factor V Leiden, increased thrombin generation, impaired fibrinolytic potential, or proteins S-C inhibition, decreased activity of antithrombin and others, are included..$^{13}$

\section{Factor V Leiden mutation}

Factor V Leiden (FVL) mutation is a moderate cause of inherited thrombophilia, accounting for 40$50 \%$ of cases. FVL mutation involves $\mathrm{G}$ (guanine) to A (adenine) substitution at nucleotide 1691 (G1691A). It accounts for $92 \%$ of cases of activated protein C resistance (APC-R). Therefore, the terms factor V Leiden and APC-R should be considered synonymous. APC-R caused by factor V Leiden mutation is the most common predisposition to hypercoagulability in white populations, mainly favoring deep venous thrombosis (DVT), venous thromboembolism (VTE) and pulmonary embolism (PE). ${ }^{14,15}$ This disorder also seldom causes the formation of clots in the arteries, inducing

Table 1. Factors inducing inherited thrombophilia.

\begin{tabular}{l}
\hline Inherited thrombophilic factors \\
\hline - Inherited hyperhomocysteine \\
- Factor V Leiden mutation \\
- Prothrombin G202 10A mutation \\
\hline - Protein C deficiency \\
- Protein S deficiency \\
- Antithrombin III deficiency \\
- A high concentration of factor VIII \\
- Dysfibrinogenemia
\end{tabular}

transient ischemic attack, or coronary events. Individuals with FVL heterozygous mutation have a risk of hypercoagulability increased by 3-4 fold, while homozygous mutation increases the risk approximately by 80 fold. Nevertheless, the heterozygous mutation is much more common than homozygous.

\section{Prothrombin G20210A}

Activated prothrombin is changed into thrombin, that converts fibrinogen into fibrin inducing the blood clot formation. It must be added that thrombin, apart with fibrinogen, reacts with calcium ions activating factor VIII, which stabilizes the fibrin-clots. ${ }^{16}$

Prothrombin-related thrombophilia is characterized by VTE, which manifests most commonly in adults as DVT in the legs or PE. The most common mutation of this protein is the prothrombin gene mutation (PTG) at nucleotide 20210 (G20210A), which represents the second most common inherited predisposition to hypercoagulability. People having a mutation in the prothrombin gene produces more prothrombin than usual. This mutation can be associated with both arterial and venous thromboembolism, mainly due to DVT and PE. Blood clots may also happen in unusual sites (such as the mesenteric or cerebral sinus vein), while coronary attacks or stroke represent two rare events. The mutation may be both homozygous and heterozygous.

\section{Natural anticoagulants deficiencies}

Deficiencies of protein $\mathrm{C}$, protein $\mathrm{S}$, or antithrombin III are important risk factors for venous thrombosis, often happening in young patients. ${ }^{17}$ The deficiencies of these natural anticoagulants are rather rare events in the general population and, combined, are found in less than $15 \%$ of all individuals. People born with a deficiency of one of the abnormal genes from either mother or father are heterozygous for this gene (more frequent). Conversely, patients can inherit the abnormal gene from both parents, and in this case, they are homozygous (more rarely occurs). As already affirmed, the prevalence of these deficiencies is associated with an increased risk of blood clots in veins, whereas that seems to play a little or no role in the blood clots in the arteries. ${ }^{18}$ Specifically, the mechanism of deficiencies of activated $\mathrm{C} / \mathrm{S}$ proteins induces venous thrombosis. It consists in the inhibition of some co-factors (factor Va, factor VIIIa) acting as a risk factor in the coagulative cascade, while a deficiency in antithrombin III acts by inhibiting the serine proteases (factor III, X, XI, XII).

\section{Elevation of factor VIII}

Several studies have pointed out an association between elevated factor VIII and increased risk of 
thrombophilia. This condition is due to the increased thrombin generation responsible for venous clots formation, often inducing thromboembolic events. ${ }^{19,20}$ Specifically, an excess of this factor resulted to be associated with a 6-fold increase of thrombo-embolic accidents.

\section{Dys-fibrinogenemia}

Defects of fibrinogenemia might be associated with increased risk for both hemorrhagic and thrombotic defects. Dysfibrinogenemia is a heterogeneous group of fibrinogen defects that may be congenital or acquired. Congenital dysfibrinogenemia is a relatively rare condition where an abnormality in the fibrin molecule results in defective clot formation. Although the exact mechanism of thrombosis is unknown, it may be related to increased fibrin formation and impaired fibrinolysis. ${ }^{21,22}$

In Table 2, the degrees of risk of inherited thrombophilia are reported.

\section{Acquired thrombophilia}

Acquired thrombophilia is associated with an increased risk of VTE and arterial thrombosis. The leading causes of acquired thrombophilia are listed in Table 3 .

Some of these are only described, such as antiphospholipid syndrome (APS), pregnancy, advanced age, heparin-induced thrombocytopenia (HIT), human immunodeficiency virus (HIV), some malignancies and oral contraceptive use. ${ }^{23}$

\section{Antiphospholipid syndrome}

Antiphospholipid syndrome is the most common form of acquired thrombophilia, mostly present in young women. It can be defined as a hypercoagulable state characterized by recurrent by venous and/or arterial thrombosis and/or pregnancy complications of fetal loss, pre-eclampsia, or eclampsia. APS was firstly described in the setting of systemic lupus erythematous, and subsequently recognized as an independent condition and in conjunction with a variety of other autoimmune infections, and malignant illnesses. ${ }^{24,25}$

The spectrum of thrombosis in APS includes both venous and arterial events, even though the mechanisms of thrombosis are highly heterogeneous, multifactorial, and not yet defined. ${ }^{26}$ One hypothesis for arterial thrombosis is that antibodies associated with APS have procoagulant effects. Other proposed mechanisms include the following: activation of platelets to enhance endothelial adherence; activation of vascular endothelium, which facilitates the binding of platelets and monocytes; the reaction of antibodies to oxidized low-density lipoproteins, and interference with the coagulation cascade. The most common venous event is DVT and spontaneous abortion or unexplained fetal death in women. ${ }^{27}$

\section{Pregnancy}

During pregnancy, clotting factors I, VII, VIII, IX, and $\mathrm{X}$ rise; protein $\mathrm{S}$ and fibrinolytic activity diminish, and resistance to activate protein $\mathrm{C}$ develops. These conditions increase the risk of venous and arterial thrombosis. The hypercoagulable state during pregnancy may increase the risk of thromboembolism. ${ }^{28}$

\section{Advanced age}

Conventional risk factors in the elderly can be acquired (immobility, surgery, malignant diseases, hor-

Table 2. Risk degrees of thrombophilia related to the inherited factors.

\begin{tabular}{l}
\hline Degrees risk of inherited thrombophilia \\
\hline High risk of thrombophilia \\
- Antithrombin deficiency \\
- Protein C deficiency \\
- Protein S deficiency \\
\hline Moderate risk of thrombophilia \\
- Factor V Leiden \\
- Prothrombin gene mutation \\
- Factor VIII \\
\hline Low risk of thrombophilia \\
- Factor IX \\
- Factor XI \\
- Hyperhomocysteinemia
\end{tabular}

Table 3. The most common causes favoring acquired thrombophilia.

\begin{tabular}{l} 
Causes of acquired thrombophilia \\
\hline *Antiphospholipid syndrome \\
\hline *Pregnancy \\
\hline *Advanced age \\
\hline Trauma \\
\hline Immobility \\
\hline *Oral contraceptive pills \\
\hline Surgery \\
*Some malignancies \\
\hline *Heparin-induced thrombocytopenia \\
\hline Myeloproliferative disorders \\
*Human immunodeficiency virus \\
*The different forms described in the text are marked with an asterisk. \\
ine 2020; 14:1296]
\end{tabular}


mone use), whereas the most well-known genetic riskfactors are deficiencies of natural anticoagulants protein $\mathrm{C}$, protein $\mathrm{S}$, antithrombin and the factor $\mathrm{V}$ Leiden and prothrombin 20210A mutations. An evident and common coagulative disorder with advancing age is also an elevated Factor VIII, associated with an approximately 5-to-7 fold increased risk of VTE. But, this increase also depends on more elevated levels of Factor IX and Factor X. ${ }^{29}$

\section{Malignancy}

Multiple prothrombotic pathogenic processes are involved in some malignancies, including activation of the coagulation cascade and fibrinolytic system, procoagulant activation of tumor cells, and tumor cell interaction with blood cells and endothelium. The incidence of thrombotic events in cancer patients increased in comparison with the normal population. Among acquired risk factors for thrombophilia in cancer, the coagulative tendency is common, especially in lymphoproliferative diseases. Polycythemia vera is a chronic myeloproliferative syndrome in which the tendency of thrombus formation is increased. The occurrence of a central venous catheter in cancer-patients is another frequent cause of thrombosis..$^{30}$ However, some inherited, thrombophilic factors also may act. Mainly, factor V Leiden or G20210A prothrombin gene mutation can be present and can increase the risk of venous thromboembolism by about 2-to-4 fold.

\section{Oral contraceptives}

Oral contraceptive pills in women can increase the risk of thrombosis. ${ }^{31}$ Among the hereditary types of thrombophilia, a resistance to activate protein $\mathrm{C}$ represents nearly $50 \%$ of cases, while in 15 to $20 \%$ a deficiency of antithrombin III, protein $\mathrm{C}$, or protein $\mathrm{S}$ is found. In addition, antiphospholipid antibodies represent a significant cause of acquired thrombophilia. They also increase during treatment with oral contraceptives and therefore represent an adjunctive thrombotic risk-factor. ${ }^{32}$

\section{Heparin-induced thrombocytopenia}

Heparin-induced thrombocytopenia consists in the development of thrombocytopenia due to the administration of various forms of heparin, an anticoagulant. HIT predisposes to thrombosis because platelets release microparticles that activate thrombin, leading to thrombosis. Specifically, HIT is caused by the formation of abnormal antibodies that increases in individuals the risk of both venous and arterial thromboses. ${ }^{33}$ Diagnosis of HIT is based on clinical features, including a decline in the platelets count $</=150,000 \mu \mathrm{L}$ or by $50 \%$ from baseline. These antibodies are directed toward antigens present on platelets. The decline in platelet count is seen between 5 and 14 days after inhibition of heparin. HIT occurs with unfractionated heparin more commonly than with low-molecularweight heparin. The risk of HIT increased in surgical patients (orthopedic and cardiac surgery). ${ }^{34}$

\section{Human immunodeficiency virus}

Another disease that induces acquired thrombophilia is human immunodeficiency virus (HIV) although the exact mechanisms for thromboembolic risk are unknown. These include the presence of antiphospholipid-anticardiolipin antibodies, decreased activity of natural anticoagulants (especially protein $\mathrm{S}$ ), and increase platelets' activation. A hypercoagulable state, including myocardial infarction, emerges in HIV-infected patients.

It has been proposed that these changes are the result of disseminated intravascular coagulopathy and endothelial injury. It must be remembered that protein $\mathrm{C}$ and $\mathrm{S}$ deficiency and APS induce 6-fold increase in the risk of VTE. Other factors, such as an increased tissue factor expression may correlate with an increased risk of VTE. ${ }^{35,36}$

\section{Therapy}

\section{Antiplatelet therapy}

To prevent recurrent arterial thrombotic events related to a hypercoagulable state, predisposed individuals and patients that have already suffered from a first episode of arterial thrombosis must be treated with antiplatelet drugs indefinitely. ${ }^{37}$

\section{Anticoagulant therapy}

Current guidelines recommend that patients who have already suffered from a previous venous thromboembolism must receive anticoagulation therapy for a minimum of three months. Those who have a family history of venous thrombosis could receive anticoagulant treatment indefinitely. This therapy may also be continued indefinitely in patients who suffered from two or more spontaneous thrombosis, in those who had one spontaneous venous thrombosis at an unusual site, and in patients who suffered from one episode in the presence of single and/or multiple genetic coagulative defects. $^{38,39}$

The anticoagulant therapy for the prevention of recurrent VTE must be started through an initial treatment with low-molecular-weight heparin followed by an oral VKA for long-term anticoagulation. Index of normalized ratio (INR) must be routinely defined, and it is crucial to perform doses-adjustments in order to maintain the INR value between 2.5-3.0. INR must be maintained persistently below these values in patients 
with an increased risk of bleeding. These INR values must also be maintained in elderly patients, in patients with renal impairment, in those having low body weight, and a history of gastrointestinal bleeding.

\section{Direct oral anticoagulants}

But, in recent years, direct oral anticoagulants, and oral non-vitamin $\mathrm{K}$ anticoagulants, are increasingly used instead of VKAs in the prevention of recurrent VTE including that associated with thrombophilia. ${ }^{40}$ DOACs offer numerous advantages over VKAs, such as a predictable response, fewer drugs, and food interactions and no need for laboratory monitoring of the INR or other coagulation tests. ${ }^{41}$

Dabigatran, rivaroxaban, apixaban, and edoxaban have been developed as a treatment alternative to heparins/VKAs. Several clinical trials found that new oral anticoagulants (NOACs) have non-inferior efficacy than heparins/VKA for both acute treatment and prevention of recurrent VTE. ${ }^{42-44}$ NOACs were till now approved for the treatment of acute DVT, PE, and the prevention of recurrent VTE. But, their use in these pathologic findings are still conflicting. ${ }^{45}$

Little is known about the efficacy and safety of DOACs in patients with venous thromboembolism in a thrombophilic pattern. Referring to hereditary thrombophilia alone, Crowther et al. classified that into two main groups, following the risk level of thrombosis.

Group 1 (also called high-risk thrombophilias), caused by hereditary deficiencies of natural anticoagulants and include protein $\mathrm{C}$, protein $\mathrm{S}$, and antithrombin deficiencies.

Group 2 (also called minor or low-risk thrombophilias), associated with increased levels of the coagulation factors and include Factor V Leiden, G20210A prothrombin mutations, an elevated level of factor VIII, IX, XI and HHcy. ${ }^{22}$ The most common thrombophilias are induced by Factor V Leiden and G20210A prothrombin with a prevalent heterozygosity (low-risk factors) ${ }^{46-48}$ In these patients, the results were favorable. On the contrary, the results obtained in protein $\mathrm{C}$ and $\mathrm{S}$ deficiency remain uncertain. APS is an acquired thrombophilic disorder associated with increased risk for venous and arterial thrombosis. DOACs treatment in APS supplied conflicting results. In fact, in RAPS (Rivaroxaban in Anti-Phospholipid Study), no thrombotic or significant bleeding was found after 210 days of follow-up, in the group that received Rivaroxaban in comparison with the patients treated with warfarin. ${ }^{49}$ Contrarily, the TRAPS (Trial of Rivaroxaban in Anti-Phospholipid Syndrome) study, designed to evaluate the efficacy of Rivaroxaban versus warfarin in APS patients, was prematurely stopped because an interim analysis showed a higher rate of thromboembolic and frequent bleeding events among the patients receiving DOAC (rivaroxaban). ${ }^{50}$

Finally, Elsebaie et al. in a systematic review of 10 studies performed on thrombophilic patients, affirmed that rates of VTE recurrence and bleeding events were comparable in patients with venous thrombophilias receiving both VKA or DOACs. ${ }^{51}$

\section{Conclusive remarks}

In conclusion, little is still known about the efficacy and safety of DOACs in patients with different forms of inherited or acquired thrombophilia. For this reason, the unlimited use of DOACs in these patients is now controversial. However, some evidence indicates that DOACs are a valuable and promising therapeutic option in therapy of VTE and its complications (TVP, PE), as well as in the secondary prevention of thrombotic events in individuals suffering from some thrombophilias. Nevertheless, other experiences performed in a wide range are requested to demonstrate their efficacy in all patients with thrombophilia clearly. However, the observations reported seem to support that DOACs are useful in low-risk thrombophilia. Their efficacy and safety appear to be insufficient in thrombophilias by protein $\mathrm{C}$, protein $\mathrm{S}$, and antithrombin deficiency (high-risk thrombophilias), while the use of these drugs is uncertain in APS patients and those with a history of arterial thrombi.

\section{References}

1. Gale AJ. Current understanding of hemostasis. Toxicol Pathol 2011;39:273-80.

2. Revel-Vilk SR, Rand ML, Israel SJ. Primary and secondary hemostasis, regulators of coagulation, and fibrinolysis: understanding the basic. In: Blanchette VR, Breakey VR, Revel-Vilk SR, eds. SickKids. Handbook of Pediatric Thrombosis and Hemostasis. Basel: Karger AG; 2013. Chapter 2, pp. 5-13.

3. Schved JF. Definition of thrombophilia. Ann Med Int 2003; 154:279-82.

4. Rambaldi MP, Mencacci F, Guaschino S. Inherited and acquired thrombophilia. Reprod Sci 2014;21:167-72.

5. Armstrong EM, Bellone JM, Hornsty LB. Acquired thrombophilia. J Pharm Prat 2014;3:234-42.

6. Previtali E, Bucciarelli P, Passamonti SM, et al. Risk factors for venous and arterial thrombosis. Blood Trans 2011;9:120-38.

7. Khan S, Dickerman JD. Hereditary thrombophilia. Thromb J 2006;4:15.

8. Girolami A, Simioni P, Scarano L, et al. Venous and arterial thrombophilia. Hematologica 1997;82:96-100.

9. Bagot CN, Arya R. Virchow and his triad: a question of attribution. Br J Hematol 2008;143:180-90.

10. Key NS, McGlennen RC. Hyperomocysteinemia and thrombophilia. Arch Pathol Lab Med 2002;126: 1367-75.

11. Brustolin S, Giugliani R, Felix TM. Genetics of homo- 
cysteine metabolism and associated disorders. Braz J Med Biol Res 2010;43:1-7.

12. Ray JC, Shmorgun D, Chan WS. Common C677T polymorphism of the methylenetetrahydrofolate reductase gene and the risk of venous thromboembolism: metaanalysis of 31 studies. Pathophysiol Hemost Thromb 2002;32:51-8.

13. Himerova J. Homocysteine and venous thromboembolism-is there any link? Cor Vasa 2013;55:E248-58.

14. Ornstein DL, Cushman M. Factor V Leiden. Circulation 2003;107:e94-7.

15. De Stefano V, Chiusolo P, Paciaroni K, et al. Epidemiology of factor V Leiden: Clinical indications. Semin Thromb Hemost 1998;24:367-79.

16. Varga EA, Moll S. Cardiology patient pages. Prothrombin 20210 mutation (factor II mutation). Circulation 2004;110:15-8.

17. Bertram B, Remky A, Arend O, et al. Protein C, Protein $\mathrm{S}$ and Antithrombin III in acute vascular occlusive diseases. Ger J Ophtalmol 1995;4:332-5.

18. Lipe B, Orstein DL. Deficiencies of natural anticoagulant, protein $\mathrm{C}$, protein $\mathrm{S}$, and antithrombin. Circulation 2011;124:e365-8.

19. O’Donnell J, Mumford AD, Manning RA, et al. Evaluation of factor VIII:C in venous thromboembolism is persistent and independent of the acute phase response. Thromb Haemost 2000;83:465-9.

20. Jenkins PV, Rawley O, Smith OP, et al. Elevated factor VIII levels and risk for venous thromboembolism. Br J Hematol 2012;157:653-63.

21. Haves T. Dysfibrinogenemia and thrombosis. Arch Pathol Lab Med 2002;126:1387-90.

22. Crowther MA, Kelton JG. Congenital thrombophilic states associated with venous thrombosis: a qualitative overview and proposed classification system. Ann Intern Med 2003;138:128-34.

23. Armstrong EM, Bellone JM, Horushy LB, et al. Acquired thrombophilia. J Pharmacy Prat 2014;27:234-42.

24. Giannakopoulos B, Krilis SA. The pathogenesis of the antiphospholipid syndrome. NEJM 2013;368:1033-44.

25. Negrini S, Pappalardo F, Murdaca G, et al. Antiphospholipid syndrome: from pathophysiology to treatment. Clin Exp Med 2017;17:257-67.

26. Grossman JM. Primary versus secondary antiphospholipid syndrome: is this lupus or not? Curr Rheumatol Rep2004;6:445-50.

27. Ronhey RA. Antiphospholipid syndrome: antibodies and antigens. Curr Opin Hematol 2000;7:316-20.Kujovich JL. Thrombophilia and pregnancy complications. Am J Obset Gynecol 2004;191:412-24.

29. Ageno W, Agnelli G, Imberti D, et al. Risk factors for venous thromboembolism in the elderly: results of master registry. Blood Coagul Fibrinolysis 2008;19:663-7.

30. Horowitz N, Brenner B. Thrombophilia and cancer. Pathol Hemost Thromb 2007;36,131-6.

31. Rosing J, Curvers J, Tans G. Oral contraceptives, thrombosis, hemostasis. Eur J Obst Gynecol Repol Biol 2001;95:193-7.

32. Bauersachs R, Kuhl H, Linhdt-Last E, Ehrly AM. Risk of thrombosis with oral contraceptives: value of thrombophilia screening test. Vasa 1996;25:209-20.

33. Linkins LA, Dans AL, Moores KL, et al. Treatment and prevention of heparin-induced thrombocytopenia: an- tithrombotic therapy and prevention of thrombosis, 9th edition. American College of Chest Physicians evidence-based clinical practice guidelines. Chest 2012;14:e495S-e530S.

34. Lindhoff-Last E, Nakov R, Misselwitz F, et al. Incidence and clinical relevance of heparin-induced antibodies in patients with deep vein thrombosis treated with unfractionated heparin and low molecular-weight heparin. $\mathrm{Br}$ J Hematol 2002;118,1137-42.

35. Erbe M, Rickezts V, Bauersachs RM, et al. Acquired protein $\mathrm{C}$ and protein $\mathrm{S}$ deficiency in HIV-infected patients. Clin Appl Thromb Hemost 2003;9:325-31.

36. Crum-Cianflone NF, Weekes J, Bavaro M. Review: thrombosis among HIV-infected patients during the highly active antiretroviral therapy era. Care STDS 2008;22:771-8.

37. Hankey GJ. Elkelboom JW, Yi Q, et al. Antiplatelet therapy and the effects of B vitamins in patients with previous stroke or transient ischemic attack: a post-hoc sub-analysis of a VITATOPS, a randomized, placebocontrolled trial. Lancet-Neurol 2012;11:512-20.

38. Kearon C, Akl EA, Comerota AJ, et al. American College of Chest Physicians. Antithrombotic therapy for VTE disease: antithrombotic therapy and prevention of thrombosis, 9th ed. American College of Chest Physicians evidence-based clinical practice guidelines. Chest 2012;141:e419S-96S.

39. Konstantinides SV, Torbicki A, Agnelli G. et al. and Task Force for the Diagnosis and Management of Acute Pulmonary Embolism of the European Society of Cardiology (ESC): 2014 ESC Guidelines on the diagnosis and management of acute pulmonary embolism. Eur Heart J 2014;35:3033-69.

40. Elkelboom JW, Weitz JL. New anticoagulants. Circulation 2010;121:1523-32.

41. Mekaj YH, Mekaj AY, Ouci SB, et al. New oral anticoagulants: their advantages and disadvantages compared with vitamin-K antagonists in the prevention and treatment of patients with thromboembolic events. Ther Clin Risk Manag 2015;11:967-77.

42. The EINSTEIN Investigators. Oral rivaroxaban for symptomatic venous thromboembolism. N Engl J Med 2010;363:2499-510.

43. Schulman S, Kearon C, Kakkar AK, et al. Dabigatran versus warfarin in the treatment of acute venous thromboembolism. N Engl J Med 2009;361:2342-52.

44. The Hokusai-VTE Investigators. Edoxaban versus warfarin for the treatment of symptomatic venous thromboembolism. N Engl J Med 2013;369:1406-15.

45. Kaklos SK, Kirkilesis GI, Tsolakis IA. Editor's Choiceefficacy and safety of the new oral anticoagulants dabigatran, rivaroxaban, apixaban, and edoxaban in the treatment and secondary prevention of venous thromboembolism: a systematic review and meta-analysis of phase III trials. Eur J Endovasc Surg 2014;48:565-75.

46. Kunk PR, Brown J, McShane M, et al. Direct oral anticoagulants in hypercoagulable states. J Thromb Thrombolysis 2017;43:79-85.

47. Petrikov AS, Shoikhet IN, Dudin DV, Karbyshev IA. Use a thrombin inhibitor for treatment of deep venous thrombosis and pulmonary thromboembolism in patients with thrombophilia. Angiol Sosud Khir 2017;23:33-40.

48. McBride A, Din R, Krishnadasan R, et al. Evaluation of 
direct oral anticoagulants in the treatment of venous thromboembolism for inherited thrombophilia disorders. Blood 2016;128:5007.

49. Cohen H, Hunt BJ, Efthymiou M, et al. Rivaroxaban versus warfarin to treat patients with thrombotic antiphospholipid syndrome, with or without systemic lupus erythematous (RAPS): a randomized, controlled, open-label, phase $2 / 3$, non inferiority trial. Lancet
Hematol 2016;3;26-436.

50. Penco V, Denas G, Zoppellaro G, et al. Rivaroxaban vs warfarin in high-risk patients with antiphospholipid syndrome. Blood 2018;132:1365-71.

51. Elsebaie MA, Van Es N, Langston A, et al. Direct oral anticoagulants in patients with venous thrombophilia: a systematic review and meta-analysis. J Thromb Hemost 2019;17:645-56. 